\title{
¿CUÁNTO TIENE LA "PREDIABETES" DE PRE-DIABETES?
}

Días atrás, mientras hacía una búsqueda bibliográfica, llamó mi atención el título de un artículo publicado en 1994 en el New England Journal of Medicine, que rezaba: "El último hombre sano"1. Su autor, Clifton W. Meador, pediatra, catedrático, investigador y prolífico escritor, relata en el mismo la complicada historia de la última persona sana; en realidad, se trata de un sujeto totalmente asintomático pero obsesionado con las enfermedades y que por ello se somete a todo tipo de evaluaciones que, sorprendentemente, siempre arrojan resultados normales. El artículo comienza con una cita, poniéndola en boca de un alumno de medicina que, al ser interrogado sobre la definición de una persona sana, responde: "Una persona sana es un paciente insuficientemente estudiado". "Dejad toda esperanza, vosotros que entráis a navegar en la red"... Internet es, al mismo tiempo, un pasaporte a la libertad y una prisión inescapable. Encontrarme con esa jocosa definición de persona sana, una humorada que muchos de nosotros utilizamos de vez en cuando, escrita en un artículo del New England, despertó en mí la compulsiva necesidad de buscar su origen, de comenzar un periplo de incierta duración y destino. Afortunadamente, el viaje fue a la vez breve y exitoso, ya que muy pronto llegué a un pueblito de Francia donde el recién instalado Doctor Knock (tal el nombre del personaje de la obra teatral de Jules Romains) $)^{2}$ pronunciaba esas palabras como parte de una ingeniosa estrategia para aumentar su clientela, atribuyéndolas nada menos que a Claude Bernard. Aliado con el pregonero, el maestro y el farmacéutico, lograba -aprovechando el natural temor de las personas ante la enfermedad y la muerte- introducir la medicina (en realidad, la pesquisa infundada de la enfermedad, la exageración de cambios totalmente triviales y la creencia de sufrir males inexistentes) en todos los rincones del otrora idílico pueblo y en cada resquicio de la condición humana de sus habitantes. Tan acertada es la sátira que es hoy reconocida por muchas fuentes como una auténtica profecía de la medicalización de la vida cotidiana.

Pero vamos al meollo del asunto. Desde hace más de medio siglo los niveles glucémicos situados entre valores normales y aquellos que per- miten diagnosticar diabetes mellitus han recibido nombres cambiantes. En este sentido, a fines de la década de 1950 Conn y Fajans ${ }^{3}$ hablaron de diabetes química y diabetes latente, dejando el término prediabetes para aquellos individuos con glucemias en todo normales pero que tenían antecedentes familiares y/o algún otro factor de riesgo para desarrollar diabetes mellitus. En 1965, el Comité de Expertos de la $\mathrm{OMS}^{4}$ recomendó el uso del término "borderline diabetes", y en 1979 el National Diabetes Data Group ${ }^{5}$ definió la categoría de tolerancia alterada a la glucosa ("impaired glucose tolerance", IGT oTAG), incorporada un año después en la clasificación de la $\mathrm{OMS}^{6}$.

Esta terminología fue introducida intencionalmente por los autores ${ }^{5}$ para aliviar el peso de la palabra "prediabetes" que podía ser vivida por los sujetos incluidos en esta denominación (y tenida en cuenta por la sociedad a su alrededor -convivientes, empleadores, aseguradoras-) como un estigma. Podemos dudar de muchas cosas en relación al término prediabetes pero existe acuerdo general en que no se trata de una debilidad o falta de firmeza, yendo a la etimología de la palabra enfermedad (in firmitas), sino que este mero dato bioquímico involucra un estado de riesgo, una situación metabólica que puede persistir o retrogradar hacia la normalidad o evolucionar hacia un cuadro nosológico más o menos bien definido (la diabetes mellitus tipo 2) y que señala asimismo una mayor probabilidad de sufrir, a futuro, enfermedad cardiovascular. Esto junto a muchos otros factores de riesgo que generalmente coexisten en el mismo individuo y que tendrían mucho más peso que la hiperglucemia leve en la generación de "eventos duros", como fue demostrado en un subgrupo de aproximadamente 3 mil pacientes con IGT/TAG o DM incluidos en la encuesta NHANES III.

Casi 20 años después, un nuevo comité de expertos convocados por la Asociación Norteamericana de Diabetes (ADA) lanza al ruedo una nueva clasificación de la diabetes mellitus ${ }^{8}$ (aún vigente, sorprendentemente), establece nuevas cifras para delimitar diabetes e IGT/TAG y crea la categoría de glucemia alterada en ayunas (" impaired fasting glucose", IFG o GAA) en un intento (exitoso, por cierto) 
de desterrar la realización de la prueba de tolerancia oral a la glucosa (PTOG) considerada hasta ese momento (y aún ahora) la mejor herramienta bioquímica para el diagnóstico de la diabetes mellitus. Sin mostrar tanto encono contra la PTOG, la OMS adoptó esta recomendación en $1999^{9}$. Pero la dirección del viento había cambiado radicalmente: el grupo de notables de la ADA recomendó deliberadamente el uso del término prediabetes (que incluía ambas categorías, la naciente IFG/GAA y la IGT/TAG que ellos mismos erróneamente condenaron a muerte) a fin de resaltar la (aparente) elevada probabilidad de enfermedad a futuro $y$, por lo tanto, la impostergable necesidad de tomar medidas al respecto.

Estos vientos siguieron soplando al comenzar el nuevo siglo y con renovados bríos: en 2003 los expertos de la ADA fijaron el límite para la glucemia normal en $99 \mathrm{mg} / \mathrm{dll}^{10}$, ampliando de esta manera la cantidad de sujetos con la espada de Damocles de la diabetes pendiendo sobre sus cabezas (o sea, con prediabetes) en forma considerable ${ }^{11}$, y por otro lado disminuyendo el valor predictivo de la categoría tanto para la aparición de diabetes como de enfermedad cardiovascular ${ }^{12}$. Tomando en cuenta estas evidencias y muchas otras, la OMS decidió conservar los límites de 110-125 mg/dl para la categoría de IFG/GAA desaconsejando además el uso del término de prediabetes y rescatando el papel fundamental de la PTOG en el diagnóstico de las alteraciones glucémicas en aquellos sujetos con glucemias en ayunas $<110 \mathrm{mg} / \mathrm{dl}^{13}$.

La incorporación de la hemoglobina glicosilada ( $\mathrm{HbA1c}$ ) al panel diagnóstico de la diabetes mellitus por la ADA aporta, a fines de la última década y principios de la actual, una nueva vuelta de tuerca al problema ${ }^{14}$. El uso de esta herramienta diagnóstica ha generado discusión y numerosos autores refutan su utilidad y conveniencia, y no sólo por los inconvenientes metodológicos o de acceso, sino por su falta de precisión en poblaciones especiales, en estadios iniciales de la diabetes y en el diagnóstico de los estados intermedios de hiperglucemia ${ }^{15}$.

Para tener una idea cabal del problema planteado por la existencia de tres criterios utilizados para diagnosticar "prediabetes" podemos tomar las cifras de un estudio realizado recientemente en China, en el que fueron evaluados casi 100 mil individuos ${ }^{16}$. De ellos, el 8,3\% presentó una tolerancia alterada a la glucosa (IGT/TAG) mientras que tres veces más sujetos $(27,2 \%)$ presentaron IFG/
GAA tomando en cuenta el criterio de la ADA. Esta proporción se amplió a 35,4\% si se incluían aqueIlos que presentaban niveles de HbA1c entre 5,7 y $6,4 \%$. Finalmente la falta de concordancia habitual entre los distintos métodos de establecer este estado de riesgo ${ }^{17}$ hizo que el $50,1 \%$ de los evaluados presentara una o más de las tres alteraciones. Extrapolando esta proporción a la población adulta china, jel número de afectados rozaría los 490 millones!

No existen, por otro lado, dudas con respecto a la probabilidad de desarrollar diabetes, complicaciones macroangiopáticas y aún microangiopáticas ${ }^{18} \mathrm{y}$ neuropáticas ${ }^{19}$ que pueden sufrir porcentajes variables de sujetos pertenecientes a las categorías de IGT/TAG y de IFG/GAA (a los que, personalmente, prefiero catalogar, como muchos otros, de portadores de glucemias alteradas en ayunas o postcarga, respectivamente). El problema reside en que utilizar una herramienta que amplía la sensibilidad a costa de una reducción en la especificidad trae aparejado un número importante (2 a 5 veces más, según los distintos estudios) de sujetos en riesgo aparente, con la consiguiente repercusión individual, social y el incremento resultante de los costos asociados a tratamientos innecesarios.

Un consenso de la Sociedad Argentina de Diabetes realizado en $2006^{20}$ puso de manifiesto la preocupación existente en nuestro medio por el aumento de falsos positivos que acompañaba al cambio propuesto por la ADA en los límites establecidos para el diagnóstico de IFG/GAA, con el consiguiente incremento en la prevalencia de sujetos catalogados como portadores de un riesgo inexistente. Consecuentemente recomendaba mantener dichos límites en los valores de 1997 (110-125 $\mathrm{mg} / \mathrm{dl}$ ) y desaconsejaba la utilización del término de prediabetes. Desde entonces hasta ahora, el cuadro no parece haber cambiado para bien, sobre todo por la inclusión de la HbA1c que conlleva una elevación de los costos del diagnóstico, sin agregar precisión al mismo. CK Meador concluía su excelente pieza de humor médico sobre el último hombre sano diciendo: "Escapar a la enfermedad en los '90 es un asunto muy serio." Por supuesto, en 2015 esto resulta mucho más difícil aún.

Pablo Arias

Médico Endocrinólogo

Prof. Titular de Fisiología Humana,

Facultad de Ciencas Médicas,

Universidad Nacional de Rosario 


\section{REFERENCIAS}

1. Meador CK. The last well person. N. Engl. J. Med. 1994; 330: 440-1.

2. Romains J. Knock o El triunfo de la Medicina. Madrid: Editorial Bruño, 1989.

3. Fajans SS, Conn JW. Prediabetes, subclinical diabetes and latent clinical diabetes: interpretation, diagnosis and treatment. En : On the nature and treatment of diabetes. Leibel BS. Wrenshall GS (editores). Excerpta Medica Int. Cong. Series 84, NewYork 1965; 641-65.

4. World Health Organization: Diabetes Mellitus: Report of a WHO Expert Committee, World Health Organisation, Geneva, 1965 (Tech. Rep. Ser., N 310).

5. National Diabetes Data Group, Classification and diagnosis of diabetes mellitus and other categories of glucose intolerance, Diabetes 1979; 28: 1039-1057.

6. World Health Organization: Expert Committee on Diabetes Mellitus, World Health Organisation, Geneva, 1980 (Tech. Rep. Ser., $N^{\circ} 646$ ).

7. Qureshi Al, Giles WH, Croft JB. Impaired glucose tolerance and the likelihood of nonfatal stroke and myocardial infarction: the Third National Health and Nutrition Examination Survey Stroke 1998; 29:1329-32.

8. The Expert Committee on the Diagnosis and Classification of Diabetes Mellitus, Report of the expert committee on the diagnosis and classification of diabetes mellitus, Diabetes Care 1997; 20: 1183-1197.

9. World Health Organization: Definition, Diagnosis and Classification of Diabetes Mellitus and its Complications: Report of a WHO Consultation. Part 1: Diagnosis and Classification of Diabetes Mellitus, World Health Organisation, Geneva, 1999.

10. The Expert Committee on the Diagnosis and Classification of Diabetes Mellitus. Follow-up Report on the Diagnosis of Diabetes Mellitus, Diabetes Care 2003; 26: 31603167.
11. Borch-Johnsen K, Colagiuri S, Balkau B, et al. Creating a pandemic of prediabetes: the proposed new diagnostic criteria for impaired fasting glycaemia. Diabetologia 2004; 47:1396-1402.

12. Forouhi NG, Balkau B, Borch-Johnsen K, et al.The threshold for diagnosing impaired fasting glucose: a position statement by the European Diabetes Epidemiology Group. Diabetologia 2006; 49: 822-827

13. World Health Organization, Definition and diagnosis of diabetes mellitus and intermediate hyperglycemia, World Health Organisation, Geneva, 2006

14. American Diabetes Association. Diagnosis and classification of diabetes mellitus. Diabetes Care. 2011; 34 (Suppl 1):S62-S69.

15. Bersoux S, Cook CB, Wu Q, et al. Hemoglobin a1c testing alone does not sufficiently identify patients with prediabetes. Am. J. Clin. Pathol. 2011; 135: 674-677

16. XuY, Wang $L, \mathrm{He} \mathrm{J}$, et al. Prevalence and control of diabetes in Chinese adults. JAMA 2013; 310:948-58.

17. James C, Bullard KM, Rolka DB, et al. Implications of alternative definitions of pre-diabetes for prevalence in US adults. Diabetes Care 2011;34: 387-91

18. Gabir M, Hanson R, Dabelea D, et al. Plasma glucose and prediction of microvacular disease and mortality: evaluation of 1997 American Diabetes Association and 1999 WHO criteria for diagnosis of diabetes. Diabetes Care 2000; 23: 1113-18.

19. Sumner C, Sheth S, Griffin J, Cornblath D, et al. The spectrum of neuropathy in diabetes and IGT. Neurology 2003; 60: 108-11

20. Gagliardino JJ, de Sereday M, González C, y cols. Conclusiones de la reunión de consenso sobre criterio diagnóstico de la glucemia de ayunas alterada de la sociedad argentina de diabetes. Rev. Soc. Arg. de Diabetes 2007; 41: 95-104. 